EPJ Web of Conferences 103, 07001 (2015)

DOI: 10.1051/epjconf/201510307001

(C) Owned by the authors, published by EDP Sciences, 2015

\title{
Rabi Oscillations Lifetime Improvement in a System of Exciton Polaritons
}

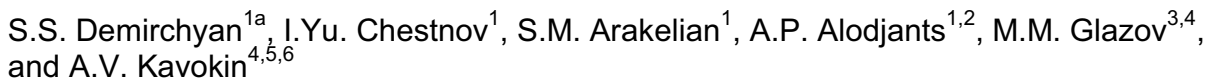 \\ ${ }^{1}$ Vladimir State University named after A.G. and N.G. Stoletov, 600000, Vladimir, Gorky str., 87, Russia \\ ${ }^{2}$ National Research University for Information Technology, Mechanics and Optics (ITMO), 197101, St. Petersburg, \\ Kronverksky Pr., 49, Russia \\ ${ }^{3}$ Ioffe Physical-Technical Institute of the RAS, 194021, St. Petersburg, Polytekhnicheskaya St., 26, Russia \\ ${ }^{4}$ Spin Optics Laboratory named after I.N. Ural'tseva, St.Petersburg State University, 198504, St. Petersburg, \\ Petrodvorets, Ulyanovskaya str., 1, Russia \\ ${ }^{5}$ Russian Quantum Center, 143025, Moscow, Skolkovo, Novaya str., 100A, Russia \\ ${ }^{6}$ School of Physics and Astronomy, University of Southampton, SO17 1NJ Southampton, UK
}

Keywords: exciton polaritons, Rabi oscillations, PT-symmetry

Polaritonics study bosonic quasiparticles, named polaritons, formed as a result of the strong lightexciton coupling. Nowadays polaritonics represents an indispensable tool for investigation of quantum coherent and nonlinear phenomena occurring at the matter-field interface. In this sense, polaritonics represents a significant interest for quantum information science. Rabi oscillations represent a natural property of exciton-polariton systems arising from the beating between the polaritons of the lower and upper branches. Usually such oscillations, being formed by external short coherent pulse, decay in time that obviously reflects a presence of decoherent processes in a system.

The decoherence time (or Rabi-oscillations lifetime) may be quite short in realistic systems, typically comparable or even shorter than the lifetime of the exciton polariton that is on the picosecond time scale. In the paper (see also [1]) we discuss the ways of creating a stable polariton Rabi-oscillations in a resonantly $\mathrm{cw}$ pumped semiconductor microcavity. We demonstrate that in the presence of an incoherent reservoir of polaritons the coherence time of Rabi oscillations may be dramatically increased (up to nanoseconds) due to stimulated scattering of polaritons towards the ground state characterized by zero in-plane wavevector. Such a research pave the way to the study of permanent Rabi-oscillations in semiconductor microcavities, when lifetime of the oscillations approach to infinity due to exciton state pumping from the reservoir. We have described the regime of permanent Rabi oscillations in a driven-dissipative exciton-photon system feeded from the incoherent exciton reservoir, cf. [2]. Two types of reservoir-Rabi-oscillator coupling mechanisms have been examined.

We have shown that the permanent Rabi oscillation regime is established above the threshold pumping where the dynamical PT-symmetry of the coupled exciton-photon system is realized.

\footnotetext{
${ }^{\mathrm{a}}$ Corresponding author: sevakdemirchyan@gmail.com
} 
Notably, this regime occurs in a non-resonant case only if the exciton component of the condensate is pumped by exciton-exciton scattering predominantly. We studied excitonic polarization properties in the presence of external magnetic fields. The conditions for permanent Rabi oscillations for polarized excitonic or photonic system are established and discussed. Realization of permanent Rabi oscillations in this case play a crucial role for creation and manipulating of long-lived spin polarization in the exciton-photon system which pave the way to the design of new optical memory devices.

\section{References}

1. S.S. Demirchyan, et al, Phys. Rev. Lett. 112, 196403 (2014)

2. I.Yu. Chestnov, et al., arXiv: 1503.07351 (2015) 